\title{
Diagnostik schlafbezogener Atmungsstörungen mittels portabler Verfahren
}

\author{
Diagnosis of Sleep Disordered Breathing Using Portable Methods
}

Autoren

Institut
T. Penzel, A. Blau, C. Garcia, C. Schöbel, M. Sebert, G. Baumann, I. Fietze

Interdisziplinäres Schlafmedizinisches Zentrum, Charité - Universitätsmedizin Berlin eingereicht 24.9 .2012
6.11.2012

akzeptiert 6.11.2012

Bibliografie

DOI http://dx.doi.org/

10.1055/s-0032-1325943

Online-Publikation: 17.12.2012

Pneumologie 2013; 67: 112-117

(c) Georg Thieme Verlag KG

Stuttgart · New York

ISSN 0934-8387

Korrespondenzadresse

Prof. Dr. Thomas Penzel

Interdisziplinäres

Schlafmedizinisches Zentrum

Charité - Universitätsmedizin

Berlin

Charitéplatz 1

10117 Berlin

thomas.penzel@charite.de

Serienherausgeber

W. Duchna, Wangen

W. Randerath, Solingen

\section{Zusammenfassung \\ $\nabla$}

Das portable Monitoring von schlafbezogenen Atmungsstörungen ist mittlerweile die Diagnostik der ersten Wahl nicht nur in Deutschland, sondern auch in anderen Ländern. Die anamnestischen und apparativen Voraussetzungen für eine verlässliche portable Diagnostik sind jetzt gut definiert. Die Grenzen und Limitationen dieser Diagnostik in Bezug auf Patientengruppen und differenzialdiagnostische Möglichkeiten sind spezifiziert. Die zur Polygrafie eingesetzten Geräte werden in vier Klassen eingeteilt, die sich an der Zahl und Art der aufgezeichneten Signale orientieren.

Neue Entwicklungen auf dem Gebiet des portablen Monitoring nutzen indirekte Möglichkeiten der Atmungsaufzeichnung, um schlafbezogene Atmungsstörungen zu erkennen. Die EKG-basierte Atmungserkennung, die plethysmografisch abgeleitete Pulswellenanalyse, die mit Magneten gemessene Kieferbewegung und Atmungsgeräuschanalysen zählen dazu. Für die neuen Verfahren liegen bis jetzt nur wenige Studien vor. Es kann sich erst mittelfristig zeigen, welche dieser Verfahren sich in der Diagnostik bewähren und welche Rolle den Systemen dann zugewiesen wird.

\section{Einleitung}

\section{$\nabla$}

Schlafbezogene Atmungsstörungen haben eine hohe Prävalenz und stellen einen inzwischen anerkannten Risikofaktor für kardiovaskuläre Folgeerkrankungen dar. Eine erfolgreiche Therapie der schlafbezogenen Atmungsstörungen senkt das Risiko, kardiovaskuläre Folgeerkrankungen zu erleiden [1]. Daher müssen schlafbezogene Atmungsstörungen rechtzeitig erkannt und auch therapiert werden [2]. Wo nun genau die Grenzen für eine empfohlene und auch für eine dringend notwendige Therapie liegen, ist noch nicht abschließend untersucht. Gesundheitsökonomische

\section{Abstract \\ $\nabla$}

Portable monitoring of sleep disordered breathing is the first diagnostic method not only in Germany but today in other countries as well. The conditions under which portable monitoring can be done with reliable results are now well defined. The limitations for the use of portable monitoring are specified as well. The devices used for portable monitoring are classified in four categories according to the number and the kind of signals recorded.

New technical developments in the field of portable monitoring (polygraphy) use an indirect assessment of sleep disordered breathing based on signals not directly recording respiration. The recording of ECG and deriving respiration, the analysis of the plethysmographically recorded pulse wave, the recording of jaw movements using magnets, and advanced analysis of respiratory sounds are recent approaches. These new methods are presented with few studies until now. More and larger clinical studies are needed in order to show which of these systems is useful in the diagnosis of sleep disordered breathing and which are the specific strengths and weaknesses.

Überlegungen sind bei einer solchen Entscheidung ebenfalls zu berücksichtigen. Die Grenzen für eine Therapieentscheidung sind womöglich nicht allein beim zur Zeit verwendeten ApnoeHypopnoe Index (AHI) und der Schläfrigkeit zu finden, sondern liegen bei einer Zusammenschau von AHI, koprävalenten kardiovaskulären Erkrankungen und Adipositas. Der intermittierenden Hypoxie und der damit einhergehenden Reoxigenation kommt bei der Pathophysiologie der kardiovaskulären Folgeerkrankungen eine herausgehobene Bedeutung zu. Eine pathogenetische Rolle spielen die hierbei entstehenden freien Sauerstoffradikale [3]. Jede einzelne Komponente trägt 
Tab. 1 Übersicht über die Review Publikationen zur überwachten kardiorespiratorischen Polysomnografie. Das Ergebnis des evidenzbasierten Prozesses ist ein Manual zur Aufzeichnung und Auswertung des Schlafes [5].

\begin{tabular}{|c|c|c|c|c|}
\hline Studientitel & Autoren & Jahr & Studienart & Ergebnis \\
\hline $\begin{array}{l}\text { The visual scoring of sleep in } \\
\text { adults }\end{array}$ & Silber M et al. [39] & 2007 & $\begin{array}{l}\text { Metaanalyse von } 26 \text { Studien nach einer } \\
\text { Begutachtung von mehr als } 1000 \text { Studien }\end{array}$ & Schlafstadien Auswertung \\
\hline $\begin{array}{l}\text { Digital analysis and technical } \\
\text { specifications }\end{array}$ & Penzel T et al. [37] & 2007 & $\begin{array}{l}\text { Metaanalyse von } 119 \text { Studien nach einer } \\
\text { Begutachtung von } 154 \text { Studien }\end{array}$ & $\begin{array}{l}\text { Technische Empfehlungen und Hinweise } \\
\text { für eine automatische Schlafanalyse }\end{array}$ \\
\hline The scoring of arousal in sleep & Bonnet MH et al. [34] & 2007 & $\begin{array}{l}\text { Metaanalyse von } 122 \text { Studien nach einer } \\
\text { Begutachtung von } 2415 \text { Studien }\end{array}$ & Arousal-Auswertung \\
\hline $\begin{array}{l}\text { The scoring of cardiac events } \\
\text { during sleep }\end{array}$ & Caples SM et al. [35] & 2007 & $\begin{array}{l}\text { Metaanalyse von } 14 \text { Studien nach einer } \\
\text { Begutachtung von } 285 \text { Studien }\end{array}$ & EKG und kardiovaskuläre Signale \\
\hline Movements in sleep & Walters AS et al. [40] & 2007 & $\begin{array}{l}\text { Metaanalyse von } 44 \text { Studien nach einer } \\
\text { Begutachtung von } 81 \text { Studien }\end{array}$ & Bewegungsauswertung \\
\hline $\begin{array}{l}\text { The scoring of respiratory } \\
\text { events in sleep }\end{array}$ & Redline S et al. [38] & 2007 & $\begin{array}{l}\text { Metaanalyse von } 182 \text { Studien nach einer } \\
\text { Begutachtung von } 2298 \text { Studien }\end{array}$ & $\begin{array}{l}\text { Empfehlungen zur Auswertung der } \\
\text { Atmung }\end{array}$ \\
\hline $\begin{array}{l}\text { The visual scoring of sleep and } \\
\text { arousal in infants and children }\end{array}$ & $\begin{array}{l}\text { Grigg-Damberger M } \\
\text { et al. [36] }\end{array}$ & 2007 & Metaanalyse von 344 Studien & Polysomnografie bei Kindern \\
\hline
\end{tabular}

unabhängig zu einem erhöhten kardiovaskulären Risiko bei. Und daher bleiben wir momentan bei der Diagnostik schlafbezogener Atmungsstörungen beim einfachsten Maß, nämlich dem AHI und der Schläfrigkeit. Damit sind wir immerhin etwas weiter als andere Länder, die allein auf den AHI sehen und dadurch zum Teil für den Patienten sehr eigenwillige Entscheidungen treffen.

\section{Diagnostische Möglichkeiten}

$\nabla$

Die Diagnostik der schlafbezogenen Atmungsstörungen ist in Deutschland nach einer Richtlinie des gemeinsamen Bundesausschusses der Ärzte und Krankenkassen geregelt. Diese Richtlinie zur Bewertung medizinischer Untersuchungs- und Behandlungsmethoden (BUB-Richtlinie) umfasst in der Anlage A „Anerkannte Untersuchungs- und Behandlungsmethoden“ und in der Anlage B „nichtanerkannte Untersuchungs- und Behandlungsmethoden“. In der Anlage A unter Punkt 3 ist die „Polygraphie und Polysomnographie im Rahmen der Differentialdiagnostik und Therapie schlafbezogener Atmungsstörungen“ für die vertragsärztliche Versorgung geregelt [4]. Danach erfolgt die Diagnostik schlafbezogener Atmungsstörungen nach einem Stufenschema mit vier Stufen. Die erste Stufe beginnt mit einer schlafmedizinischen Anamnese, unterstützt durch validierte Fragebögen zur Einschlafneigung am Tage, zu beobachteten oder berichteten nächtlichen Atempausen und intermittierendem Schnarchen. Es folgen Untersuchungen mit BMI, Blutdruck, Körpermaßen (Hüft- und Halsumfang), zur körperlichen Belastung, um eine Verdachtsdiagnose zu erhalten. Im dritten Schritt wird eine portable Untersuchung auf schlafbezogene Atmungsstörungen durchgeführt. Bleibt das Ergebnis dieser gewöhnlich ambulant oder als Konsil durchgeführten Untersuchung unklar oder liegen weitere Erkrankungen vor, die eine Interpretation der Ergebnisse erschweren (COPD, respiratorische Insuffizienz, Herzinsuffizienz, neurologische Erkrankungen), dann muss eine Untersuchung im Schlaflabor mit überwachter kardiorespiratorischer Polysomnografie erfolgen. Das bedeutet, dass der letzte Schritt der Diagnostik im Schlaflabor einer kleineren Untergruppe vorbehalten bleibt. Es bedeutet aber auch, dass genügend Schlaflabore verfügbar sein müssen, die diesen letzten Schritt mit der vollständigen schlafmedizinischen Expertise anbieten können. Die Patienten, die zu dieser Schlaflabor-Diagnostik kommen, sind gewöhnlich Patienten mit Begleiterkrankungen, die eine aufwändige Betreuung erfordern und dadurch nicht allein eine Polysomnografie er- halten, sondern darüber hinaus Untersuchungen am Tage zu ihren Begleiterkrankungen.

\section{Kardiorespiratorische Polysomnografie}

Die Referenz für alle Untersuchungen im Schlaflabor und für portable Untersuchungen ist die kardiorespiratorische Polysomnografie mit Überwachung im zertifizierten Schlaflabor. Die Empfehlungen zur Ableitung und Aufzeichnung der physiologischen Signale des Schlafenden sind in einem internationalen Manual für die Aufzeichnung und Auswertung festgehalten [5]. Das Manual stellt eine Erweiterung einer älteren Empfehlung zur Aufzeichnung und Auswertung nur des Schlaf-EEGs dar. So sind neben Empfehlungen zum EEG, EOG, und EMG auch Empfehlungen zur Aufzeichnung der Atmung, der Sauerstoffsättigung und der Bewegung der Gliedmaßen enthalten. Die Körperlage muss auch aufgezeichnet werden, und eine Videoüberwachung mit der Möglichkeit der Aufzeichnung ist ebenfalls erforderlich. Das Manual ist das Ergebnis eines evidenzbasierten Begutachtungsprozesses der existierenden Literatur ( Tab. 1). Damit stellt diese Referenzmethode eine valide und gesicherte Diagnostik dar, die als Referenz herangezogen werden kann.

Selbst bei diesem schlafmedizinischen Standard sind noch eine Reihe von Fragen offen geblieben. So ist noch nicht geklärt, welches der zusätzliche Wert der Schlafauswertung Epoche für Epoche bei den schlafbezogenen Atmungsstörungen ist. Womöglich reicht für klinische Zwecke eine Einteilung in Wach, Leichtschlaf, Tiefschlaf und REM-Schlaf aus. Um den klinischen Wert der differenzierten Schlafparameter zu ermitteln, müssen die berechneten Größen (Schlafdauer, Schlafeffizienz, Prozente der Schlafstadien, Latenzen) in einen Zusammenhang mit klinischen Outcome-Parametern gebracht werden. Es bleibt ebenfalls offen, welche Bedeutung die Unterscheidung von Apnoen und Hypopnoen auf klinische Outcome-Parameter hat.

Bezogen auf die schlafbezogenen Atmungsstörungen sind die Pathomechanismen der Schlaffragmentation und der rezidivierenden Arousal inzwischen gut verstanden, deren klinische Bedeutung ist aber noch nicht komplett geklärt. In verschiedenen Studien wurde belegt, dass die Schlaffragmentation und die Arousal mit Schläfrigkeit am Tage einhergehen, wobei es auch keine lineare Korrelation zu geben scheint [6,7]. Ebenso ist der Zusammenhang zwischen nächtlichen Hypoxien und dem kardiovaskulären Risiko nicht ein einfacher linearer, es spielen 


\begin{tabular}{|lll|}
\hline Klasse & $\begin{array}{l}\text { Bezeichnung } \\
\text { überwachte kardiorespira- } \\
\text { torische Polysomnografie }\end{array}$ & $\begin{array}{l}\text { Charakteristika } \\
\text { Polysomnografie mit mindestens } 7 \text { Signalen. Überwachung durch geschultes } \\
\text { schlafmedizinisches Personal. Folgt den AASM Kriterien [5]. }\end{array}$ \\
\hline 2 & $\begin{array}{l}\text { kardiorespiratorische } \\
\text { Polysomnografie }\end{array}$ & $\begin{array}{l}\text { Polysomnografie mit mindestens } 7 \text { Signalen. Keine Überwachung, kann zu- } \\
\text { hause durchgeführt werden. Keine Intervention. Folgt den AASM Kriterien [5]. }\end{array}$ \\
\hline 3 & $\begin{array}{l}\text { Polygrafie/portable } \\
\text { Schlafapnoe- Diagnostik }\end{array}$ & $\begin{array}{l}\text { Polygrafie mit mindestens } 4 \text { bis } 6 \text { Signalen für Atmung, Sauerstoffsättigung, } \\
\text { Herzfrequenz, Körperlage. Nicht an einen Ort gebunden. }\end{array}$ \\
\hline 4 & Vordiagnostik, Aktigrafie & $\begin{array}{l}\text { Untersuchung mit 1 bis 3 Signalen für unterschiedliche Fragestellungen zu } \\
\text { Schlafstörungen (Langzeit-EKG, Langzeit-Blutdruck, Langzeit-EEG, Schlafap- } \\
\text { noe-Vordiagnostik, Aktigrafie). Option zum Screening. }\end{array}$ \\
\hline
\end{tabular}

Tab. 2 Klassen der portablen und der nicht-portablen Schlafdiagnostik.
Schlaffragmentation und erhöhter Sympathikus ebenfalls eine Rolle [8].

Das Favorisieren einer kardiorespiratorischen Polysomnografie bei der Diagnostik der schlafbezogenen Atmungsstörungen leitet sich genau von den noch nicht vollständig geklärten Zusammenhängen ab. Solange die Zusammenhänge zwischen dem individuellen kardiovaskulären Risiko eines Patienten und den beobachteten Parametern der Polysomnografie nicht linear sind, solange kann eine umfassende Polysomnografie dazu beitragen, das diagnostische Bild vollständig zu erfassen. Wir müssen die Schlafdauer, die Schlaffragmentation, die Arousal, die Apnoen, Hypopnoen und Hypoxien quantitativ erfassen und für die Bewertung des individuellen Risikos heranziehen.

\section{Portable Schlafapnoe-Diagnostik}

Portable Diagnostik zur Untersuchung von schlafbezogenen Atmungsstörungen ist gegenüber der überwachten kardiorespiratorischen Polysomnografie weniger aufwendig und damit kostengünstiger. Es werden weniger Signale aufgezeichnet. Die Messung zuhause belegt nicht Krankenhaus- oder andere institutionelle Betten. Die Anzahl der von einer Einrichtung eingesetzten Systeme kann dadurch höher sein und ist durch die Zeit des Anlegens und Auswertens beschränkt und nicht durch die Anzahl der verfügbaren Betten. Die portable Schlafdiagnostik wurde in vier Klassen eingeteilt ( $\checkmark$ Tab.2) [9]. Diese vier Klassen wurden in späteren Übersichtsarbeiten zu aktuellen Systemen wieder aufgegriffen $[10,11]$.

Die Klasse 1 wurde als Referenzmethode bereits weiter oben vorstellt. Die Klasse 2 entspricht vom apparativen her der Referenzmethode und ist in der Praxis Forschungsfragen vorbehalten, da der Aufwand eher noch größer als bei der überwachten Polysomnografie ist. Die Sensoren müssen nämlich so gut befestigt werden, dass sie während der Aufzeichnung nicht korrigiert oder neu befestigt werden müssen. Die Klasse 3, die Polygrafie oder portable Schlafapnoe-Diagnostik, in Deutschland häufig „Schlafapnoe-Screening“ genannt (es handelt sich nicht um ein Screening im epidemiologischen Sinn), ist die wirtschaftlich sinnvolle Diagnostik von Patienten mit schlafbezogenen Atmungsstörungen. Diese Diagnostik ist in den oben genannten BUB-Richtlinien zur Diagnostik von schlafbezogenen Atmungsstörungen vorgesehen und zugelassen [4]. Die Klasse 4 umfasst Systeme, die noch weniger Signale aufzeichnen und oft eine gute Abschätzung von schlafbezogenen Atmungsstörungen erlauben. Sie bieten für die Diagnostik der schlafbezogenen Atmungsstörungen jedoch keine ausreichende Sicherheit, um eine Therapie einzuleiten.

Ein wichtiger Aspekt in der Diskussion zur Rolle der portablen Schlafapnoe-Diagnostik und der überwachten kardiorespiratorischen Polysomnografie ist der gesundheitsökonomische Kostenaspekt. Hierzu gibt es eine Analyse von Chervin, der auf der Basis der amerikanischen Kosten für kardiorespiratorische Polysomnografie, portable Schlafapnoe-Untersuchung und auf der Basis der Diagnoseungenauigkeiten von Geräten Ende der 1990er-Jahre eine Kosten-Nutzen-Rechnung erarbeitete. Es wurden die überwachte kardiorespiratorische Polysomnografie, die portable Schlafapnoe-Diagnostik und die Therapieindikation allein auf der Basis des klinischen Verdachts in der Kosten-Nutzen-Analyse verglichen. Unter Einbeziehung des Nutzens in Form von QALYs (Quality adjusted life years) und der Kosten notwendiger sowie unnötig verordneter CPAP-Therapie ergibt sich eine ökonomische Überlegenheit der Polysomnografie [12]. Nun ist auch diese Modellrechnung an sich kritisierbar und bezüglich der Kosten nicht auf Deutschland übertragbar. Zudem hat sich die Qualität der portablen Systeme weiterentwickelt. Die Auffassung, dass die direkte Untersuchung mit kardiorespiratorischer Polysomnografie günstiger ist als erst eine vorgeschaltete Polygrafie, ist weiter vorhanden. Nur eine neue ökonomische Untersuchung in einem vorgegebenen Umfeld mit aktuellen Geräten kann hier Klarheit bringen.

In vielen Ländern und insbesondere in den USA war eine Diagnostik von schlafbezogenen Atmungsstörungen ausschließlich durch die Klasse 1 vorgesehen. Dies wurde immer wieder durch evidenzbasierte Übersichten bestätigt. Ross et al. 2000 haben im Auftrag des Health Technology Assessment (HTA) Studien mit Geräten der Klasse 3 analysiert und festgestellt, dass Sensitivität und Spezifität so schlecht waren, dass die Geräte nicht zur Diagnostik von Schlafapnoe infrage kamen [13]. Sie berichteten aus den Studien bis zu 17\% falschnegative und zwischen $2 \%$ und 31\% falschpositive Diagnosen von Schlafapnoe. Erst 2007 kam eine neue Übersicht aktueller Studien von Collop et al. zum Schluss, dass unter genau vorgegebenen Bedingungen, wie sie in den Artikeln dokumentiert wurden, doch eine sehr gute Sensitivität und Spezifität auf schlafbezogene Atmungsstörungen erzielt werden kann [14]. Diese Arbeit führte zu einem Wechsel der Diagnostik der Schlafapnoe in den USA, der jedoch noch einige Zeit in Anspruch nahm. Seit 2011 wird die Diagnostik der Schlafapnoe in den USA auch ambulant durchgeführt. Eine KonsensusKonferenz der American Thoracic Society (ATS), der American Academy of Sleep Medicine (AASM), des American College of Chest Physicians (ACCP) und der European Respiratory Society (ERS) hat die Möglichkeiten und Grenzen der portablen Schlafapnoe-Diagnostik erörtert und in Empfehlungen festgehalten [15]. Es wurden auch offene Forschungsfragen identifiziert. Dazu gehört die oben genannte gesundheitsökonomische Untersuchung zur Rolle der Polysomnografie und Polygrafie. Bei den Fragen blieb die Rolle der Klasse-4-Geräte komplett offen.

Die entscheidenden Randbedingungen, die durch Collop et al. identifiziert wurden und eine portable Diagnostik der schlafbezogenen Atmungsstörungen mit guter Sensitivität und Spezifität erlauben, lassen sich unter dem Stichwort der hohen PrätestWahrscheinlichkeit zusammenfassen [14]. In den bei dem Re- 
view herangezogenen Studien wurden immer Patienten untersucht, die schon den Weg zur Schlafmedizin gefunden hatten oder spezielle Risikogruppen darstellten. D.h. diese Patienten waren durch Beschwerden und Symptome auffällig geworden. Es handelte sich also nicht um Bevölkerungsstichproben. Bei einer solchen Vorselektion ergab sich dann mit unterschiedlichen Systemen eine hohe Sensitivität und Spezifität. Die Vorselektion lässt sich durch die folgenden Beschwerden und Symptome prozeduralisieren: (1) lautes und unregelmäßiges Schnarchen, (2) beobachtete oder berichtete nächtliche Atmungsstillstände, (3) exzessive Tagesschläfrigkeit in ungewollten Situationen, (4) unspezifische psychische Probleme wie Abgeschlagenheit, Müdigkeit, Leistungsknick, (5) überdurchschnittlich viel Bewegungen im Schlaf, Schwitzen, (6) morgendlicher Kopfschmerz und Benommenheit, trockener Mund, (7) sexuelle Funktionseinschränkungen, (8) Übergewicht, (9) arterielle Hypertonie, nächtliche Herzrhythmusstörungen.

\section{Randbedingungen portabler Diagnostik}

\section{$\nabla$}

Aus der Notwendigkeit einer hohen Prätest-Wahrscheinlichkeit haben dann Collop et al. ihre Randbedingungen hergeleitet [14]. Ihre Empfehlungen sind im Einzelnen:

(1) Systeme zum portablen Schlafapnoe-Monitoring sollen nur von zertifizierten Schlafmedizinern eingesetzt werden, die an Schlafmedizinischen Zentren tätig sind. Die Schlafmediziner können eine gezielte schlafmedizinische Anamnese erheben und dadurch die geforderte Prätest-Wahrscheinlichkeit erhöhen.

(2) Eine portable Schlafapnoe-Diagnostik wird empfohlen, wenn keine komorbide pneumologische, kardiovaskuläre, psychiatrische, neurologische Störung und keine neuromuskuläre Erkrankung, keine Herzinsuffizienz und keine andere Schlafstörung vorliegt. Andere Schlafstörungen wären zentrale Schlafapnoe, periodische Beinbewegungen im Schlaf, Insomnie, zirkadiane Schlaf-Wach-Rhythmusstörungen und Narkolepsie.

(3) Gemäß den publizierten Studien können die portablen Systeme nicht mit Sicherheit zwischen obstruktiven und zentralen Schlafapnoe-Ereignissen unterscheiden.

(4) Ambulante Systeme für die Schlafapnoe-Diagnostik müssen die folgenden Signale aufzeichnen: oronasalen Luftfluss mittels nasaler Staudrucksensoren oder Thermistoren, Atmungsbewegungen mittels Induktionsplethysmografie, Sauerstoffsättigung mit einer kurzen Pulsmittelwertbildung (über drei bis sechs Pulse), Puls- oder Herzfrequenz und Körperlage.

(5) Die Auswertung der Aufzeichnungen muss visuell erfolgen und soll den gleichen Regeln folgen, die für die Auswertung der Atmung bei der Polysomnografie gelten [5]. Ein Editieren der ausgewerteten Ereignisse ist erforderlich, um Artefakte während der Aufzeichnungsdauer zu beseitigen. Daher soll die visuelle Auswertung durch schlafmedizinisch geschultes Personal erfolgen.

(6) Die technischen Spezifikationen und die Abtastraten für die digitale Aufzeichnung bei den Polygrafie-Geräten sollen die gleichen sein, wie sie für die kardiorespiratorische Polysomnografie gelten.

Heute erfüllen zahlreiche Systeme diese Anforderungen. Alle Geräte haben ein Pulsoximeter integriert, und die meisten Geräte zeichnen den Luftfluss über nasale Staudrucksensoren auf. Die Atmungsbewegung wird bei vielen Systemen noch mit einfachen Piezosensoren abgeleitet. Jedoch bieten die meisten Systeme auch eine Induktionsplethysmografie für die Atmungsbewegungen an. Viele Systeme zeichnen nur thorakale Atmungsbewegun- gen mit einem Gürtel auf, um das Anlegen der Polygrafen zu vereinfachen bzw. ein Selbstanlegen durch den Patienten zu ermöglichen. Alle Systeme zeichnen die Körperlage auf. Nur wenige Systeme zeichnen das EKG bzw. die daraus abgeleitete Herzfrequenz auf. Die meisten Systeme zeichnen die Pulsfrequenz auf, die vom Pulsoximeter mitgeliefert wird. Diese kann Herzrhythmusstörungen nicht identifizieren. Zusätzlich bieten die meisten Systeme an, optional den CPAP bzw. den Beatmungsdruck mit aufzuzeichnen. Diese Option ist ganz wesentlich für ambulante Therapie-Kontrolluntersuchungen und sollte nicht fehlen.

Die Differenzierung von zentralen und obstruktiven Schlafapnoe-Ereignissen ist ein Thema großen Interesses, da Patienten mit Herzinsuffizienz mehr zentrale Schlafapnoe aufweisen als andere Patienten. Nun sind nach den Empfehlungen von Collop et al. Patienten mit Herzinsuffizienz explizit von einer portablen Diagnostik auf Schlafapnoe ausgenommen [14]. Es fehlen also in diesem Bereich noch Studien, die die Sensitivität und Spezifität portabler Systeme bei diesen Patienten belegen. Da viele Systeme die Atmungsbewegungen nur mit einem Gürtel aufzeichnen, ist es nicht erstaunlich, dann auch obstruktive und zentrale ApnoeEreignisse nicht ausreichend differenzieren zu können. Womöglich ist eine Untergruppe spezieller Systeme doch in der Lage, zentrale und obstruktive Apnoe-Ereignisse zu unterscheiden. Dies ist jedoch noch nicht belegt.

Einige wenige Systeme bieten an, das EMG der Beine aufzuzeichnen, um nächtliche Beinbewegungen zu diagnostizieren. Dies ist sicher eine hilfreiche Option. Jedoch fehlen noch systematische Studien zu dieser Option. Ebenso fehlen noch systematische Studien zur Option einer gleichzeitigen EKG oder Blutdruckmessung. Bezogen auf die erforderlichen technischen Spezifikationen gibt es nur noch geringe Unterschiede in Sensitivität und Spezifität der Geräte, sodass keinem System eine ausdrückliche Präferenz gegeben werden kann.

\section{Neue Systeme für portable Schlafapnoe- Untersuchungen}

Vergleichende Untersuchungen zu Systemen mit weniger Parametern, zu Systemen der Klasse 4, zeigten, dass diese nicht für eine Diagnose von Schlafapnoe geeignet sind. In $\bullet$ Tab. 2 wurden bereits das Langzeit-EKG, die Langzeit-Blutdruckmessung und die Aktigrafie genannt. Die Aktigrafie ist zur quantitativen Erfassung von Schlaf-Wachzeiten zusätzlich zu einem Schlaftagebuch sehr gut geeignet [2]. Schlafbezogene Atmungsstörungen werden durch eine Aktivitätsauswertung in einem Raster von 1-Minuten-Abschnitten nicht erfasst. Nächtliche Herzrhythmusstörungen und nächtlicher Blutdruckanstieg können gute Hinweise auf das Vorliegen von schlafbezogenen Atmungsstörungen geben. Diagnostische Instrumente für eine Schlafapnoe sind diese Systeme jedoch nicht.

Viele technische Entwicklungen versuchen mit wenigen Signalen eine gute Vorhersage auf Schlafapnoe zu erzielen. Eine möglichst gute Aufzeichnung des nasalen Luftflusses, kombiniert mit einer Pulsoximetrie, ist eine reduzierte diagnostische Möglichkeit, die ökonomisch unter Verzicht auf Redundanzen nächtliche Atmungsereignisse aufzeichnet [16]. Dabei wird versucht, den Luftfluss auch auf Schnarchen hin zu analysieren. Das charakteristische intermittierende Schnarchen und womöglich einzelne Komponenten der Schnarchgeräusche werden auf Sensitivität und Spezifität hin untersucht [17]. Die Entwicklung dieser Arbeitsgruppe basiert auf umfangreichen Erfahrungen aus der Lungen- 
geräuschanalyse. Einige Ingenieure versuchen die Auswertung der Pulsoximetrie alleine zu optimieren und aus der Pulsfrequenz die apnoespezifische zyklische Variation der Herzfrequenz zu erkennen [18]. Dies ist jedoch nicht ganz einfach, weil die Pulsfrequenz ebenso wie die Sauerstoffsättigung gewöhnlich über einige Pulschläge gemittelt ist. Die Mittelung ist bedingt durch das Verfahren, indirekt am Finger den Pulsschlag abzunehmen. Und am Finger finden sich mehr Bewegungsartefakte als bei einer direkten elektrophysiologischen EKG-Aufzeichnung.

Das EKG bietet einmal die Möglichkeit einer Erkennung von schlafbezogenen Atmungsstörungen über die zyklische Variation der Herzfrequenz. Die zyklische Variation der Herzfrequenz mit relativen Bradykardien während der Apnoephasen und relativen Tachykardien während der intermittierenden Hyperpnoe-Phasen ist eine Folge der autonomen Regulation, die mit jedem einzelnen Apnoeereignis einher geht. Sofern nicht andere Erkrankungen die Regulation der Herzfrequenz massiv beeinflussen, ist dieses Muster sehr charakteristisch für Schlafapnoe [19]. Darüber hinaus zeigt das EKG aber noch andere spezifische Veränderungen bei Schlafapnoe. Die Amplitude der R-Welle und der T-Welle ändern sich mit jedem Atemzug durch die mechanische Änderung der Herzachse bei der Atmung [20]. Es kann also aus den Änderungen der R-Wellen-Amplitude eine Atmung berechnet werden, die sogenannte „electrocardiographic derived respiration (EDR)“. Bei der obstruktiven Schlafapnoe ist diese Amplitudenschwankung durch die massiven intrathorakalen Druckschwankungen sogar noch verstärkt. Werden beide Informationen, die zyklische Variation der Herzfrequenz und die EDR, kombiniert, so lässt sich die Schlafapnoe aus dem EKG mit überraschender Genauigkeit in Bezug auf Sensitivität und Spezifität bestimmen - sofern nicht weitere kardiovaskuläre Erkrankungen vorliegen [21]. Die Kombination dieser beiden aus dem EKG berechneten Komponenten ist deswegen so stark und aussagekräftig, weil es sich um eine Kombination von zwei komplett unabhängigen Einflüssen, nämlich autonomes System und Herzmechanik, handelt. Die Herzfrequenz bietet darüber hinaus noch weitere Informationen zum autonomen System. Sie variiert mit den Schlafstadien, bezogen auf Wach, Leichtschlaf, Tiefschlaf und REM-Schlaf. Es kann also eine ungefähre Abschätzung des Schlaf-Wachrhythmus vorgenommen werden [22].

Als Konsequenz der sehr hilfreichen Informationen aus dem EKG und aus der Pulsoximetrie entstanden mehrere Studien zur Kombination beider Signale [23, 24]. Komplexe statistische Analysen des EKG und der Herzfrequenz ergeben eine hohe Zuverlässigkeit bei der Bestimmung von Apnoeereignissen [25]. Dabei werden die mit den Apnoen einhergehenden Desaturationen aus der Pulsoximetrie bestimmt und quantitativ angegeben. Und aus dem EKG wird konfirmatorisch die Atmung bestimmt und darüber hinaus auch noch eine Schlaf-Wach-Abschätzung vorgenommen [26].

Ein anderer Ansatz leitet über den peripheren Gefäßtonus den autonomen Tonus bei Schlafapnoe ab und kombiniert dieses Signal mit der Sauerstoffsättigung [27,28]. Der periphere arterielle Gefäßtonus, kombiniert mit Sauerstoffsättigung und Aktigrafie, ist als einziges Drei-Kanal-System in den USA zur frühen Diagnostik von schlafbezogenen Atmungsstörungen zugelassen. Dies liegt an den zahlreichen Validierungsstudien von unabhängigen Arbeitsgruppen, die mit guten Ergebnissen den Wert dieser indirekten Technologie im Vergleich zur Polysomnografie belegen. Eine besondere Stärke des autonomen peripheren Gefäßtonus liegt in der Erfassung der autonomen Arousal. Und daher wird die Sauerstoffsättigung mit den Entsättigungen als Korrektiv heran gezogen, um die Anzahl der abgeschätzten Schlafapnoe-Er- eignisse zu verbessern. Aus der Aktigrafie erfolgt eine Abschätzung der Schlafzeit. Und zusätzlich erfolgt aus der Variation der Pulsfrequenz eine Schätzung für REM und non-REM-Schlaf, ähnlich, wie dies auch aus der Herzfrequenz erfolgen kann.

Ein komplett anderes Verfahren untersucht die Bewegungen des Kiefers und damit indirekt des Muskeltonus zur Erfassung von Schnarchen, Schlafapnoe und Schlaf-Wachrhythmus [29,30]. Mit kleinen Magnetsensoren am Kopf und am Kiefer werden die Bewegungen des Kiefers relativ zum Schädel gemessen und analysiert. Besonders beeindruckend ist die Möglichkeit, durch das Nachlassen der Muskelspannung sogar den Einschlafvorgang zu erfassen [31]. Dieses neuartige Verfahren wurde bisher noch wenig außerhalb der erfindenden Arbeitsgruppe erprobt. Daher sind weitere Validierungen erforderlich, ehe eine definitive Bewertung vorgenommen werden kann.

Zusammengefasst kann festgehalten werden, dass zahlreiche Methoden zur indirekten diagnostischen Erfassung der Schlafapnoe entwickelt wurden und weiter entwickelt werden. So sind derzeit auch berührungslose Verfahren zur Erfassung der Bewegungen der Atmung und des Herzschlages in Entwicklung. Die modernen technischen Möglichkeiten bieten hier noch viele Optionen, die aber immer kritisch gegenüber der Referenz im Schlaflabor evaluiert werden müssen.

\section{Diskussion \\ $\nabla$}

Die kardiorespiratorische Polysomnografie ist evidenzbasiert und ausgereift zur Diagnostik von Schlafstörungen. Die Polygrafie bzw. das portable Monitoring zur Diagnostik der Schlafapnoe ist ebenfalls gut definiert, ausgereift und als diagnostisches Instrument für die Schlafapnoe unter gut angegebenen Randbedingungen evidenzbasiert geeignet. Offen bleibt an dieser Stelle die Frage nach einer quantitativen Auswertung der Prätest-Wahrscheinlichkeit. Welcher Faktor spielt welche Rolle? Lässt sich ein Algorithmus für eine Gewichtung der Prätest-Wahrscheinlichkeit erarbeiten?

Die neuen Systeme, die mit weniger Signalen auskommen, sind oft deutlich einfacher und womöglich auch kostengünstiger. Inwieweit sie geeignet sind, auch eine Diagnostik von Schlafapnoe mit guter Sensitivität und Spezifität zu erzielen, muss noch weiter in Studien evaluiert werden.

Ein Instrument zum Screenen (im epidemiologischen Sinn) auf Schlafapnoe bei Probanden ohne Beschwerden und Symptome (wie z.B. jeder Bus- oder LKW Fahrer) ist gemäß der Literatur noch nicht identifiziert und validiert. Diese Untersuchungen stehen noch aus und haben hohe Relevanz aufgrund der Konsequenz exzessiver Schläfrigkeit bei den Schlafapnoe-Patienten. Hierfür sind aufgrund des niedrigeren Aufwandes und der niedrigen Kosten die Systeme mit wenigen Signalen besonders interessant. Es ist sehr gut möglich, dass Geräte der oben genannten Klasse 4 hierfür gut geeignet sind. Für Screening-Fragestellungen muss insbesondere eine hohe Sensitivität gewährleistet sein. Gleichzeitig darf die Spezifität nicht sehr schlecht sein, da dies unnötige Kosten für weitere Untersuchungen hervorrufen würde.

Insgesamt gesehen haben wir ein sehr gutes diagnostisches Instrumentarium zur Verfügung, und basierend darauf ist heute zu überlegen, welches System welche Rolle im Prozess der schlafmedizinischen Diagnostik einnehmen kann [32,33]. Das schlafmedizinische Instrumentarium soll in der Hand schlafmedizinisch ausgebildeter Ärzte bleiben. Die Diagnostik der Schlafapnoe wird womöglich auch in den USA nicht mehr nur von schlafme- 
dizinischen Zentren durchgeführt. Diese gesundheitsökonomischen Fragen sind in Hinblick auf die Verteilung begrenzter Ressourcen heute die vordringlichen Forschungsfragen, wie auch vom Workshop zur Diagnostik schlafbezogener Atmungsstörungen festgehalten wurde [15]. Um Antworten auf diese Fragen zu finden, sind Studien in den jeweils regional gültigen Gesundheitssystemen mit den aktuellen diagnostischen Geräten und deren Validität und Reliabilität erforderlich.

\section{Interessenkonflikt}

T. Penzel hat Studienunterstützung von den Firmen Apnex Medical, Itamar, Hoffrichter, Philips/Respironics, Resmed, Somnomedics, Somnodent, Weinmann erhalten. Er ist an den Firmen Advanced Sleep Research, Somnico und The Siestagroup beteiligt. Die Autoren A. Blau, C. Garcia, C. Schöbel, M. Sebert, G. Baumann und I. Fietze geben an, dass kein Interessenkonflikt besteht.

\section{Literatur}

1 Marin JM, Carrizo SJ, Vicente $E$ et al. Longterm cardiovascular outcomes in men with obstructive sleep apnoea-hypopnoea with or without treatment with continuous positive airway pressure: an observational study. Lancet 2005; 365: 1046 - 1053

2 Mayer G, Fietze I, Fischer J et al. S3-Leitlinie Nicht erholsamer Schlaf/ Schlafstörungen. Somnologie 2009; 13: 4-160

3 Fletcher EC, Lesske J, Qian W et al. Repetitive, episodic hypoxia causes diurnal elevation of blood pressure in rats. Hypertension 1992; 19: $555-561$

4 Gemeinsamer Bundesausschuss. Beschluss über eine Änderung der Richtlinien zur Bewertung medizinischer Untersuchungs- und Behandlungsmethoden gemäß $§ 135$ Abs. 1 des Fünften Buches Sozialgesetzbuch (BUB-Richtlinien) in Anlage A „Anerkannte Untersuchungs- und Behandlungsmethoden“. Deutsches Ärzteblatt 2005; 102: A455-A459

5 Iber C, Ancoli-Israel S, Chesson A et al. for the American Academy of Sleep Medicine The AASM Manual for the Scoring of Sleep and Associated Events: Rules, Terminology and Technical Specifications. 1st ed. Westchester, Illinois: American Academy of Sleep Medicine; 2007

6 Guilleminault C, Partinen M, Quera-Salva MA et al. Determinants of daytime sleepiness in obstructive sleep apnea. Chest 1998; 94: 32 - 37

7 Rühle KH, Franke KJ, Nilius G. Sekundenschlaf, Schläfrigkeit und Verkehrstüchtigkeit beim Schlafapnoe-Syndrom. Pneumologie 2008; 62: 595-601

8 Leßke J, Fletcher EC, Bao G et al. Hypertension cause by chronic intermittent hypoxia - influence of chemoreceptors and sympathetic nervous system. J Hypertension 1997; 15: 1593-1603

9 Standards of Practice Committee of the American Sleep Disorders Association. Practice Parameters for the use of portable recording in the assessment of obstructive sleep apnea. Sleep 1994; 17: 372 - 377

10 Chesson AL, Berry RB, Pack A. Practice parameters for the use of portable monitoring devices in the investigation of suspected obstructive sleep apnea in adults. Sleep 2003; 26: $907-913$

11 Flemons WW, Littner MR, Rowley JA et al. Home diagnosis of sleep apnea: a systematic review of the literature. An evidence review cosponsored by the American Academy of Sleep Medicine, the American College of Chest Physicians, and the American Thoracic Society. Chest 2003; $124: 1543-1579$

12 Chervin RD, Murman DL, Malow BA et al. Cost-utility of three approaches to the diagnosis of sleep apnea: polysomnography, home testing, and empirical therapy. Ann Intern Med 1999; 130: 495-505

13 Ross SD, Sheinhait IA, Harrison KJ et al. Systematic review and metaanalysis of the literature regarding the diagnosis of sleep apnea. Sleep 2000; 23: 519-532

14 Collop NA, Anderson WM, Boehlecke B et al. Clinical guidelines for the use of unattended portable monitors in the diagnosis of obstructive sleep apnea in adult patients. J Clin Sleep Med 2007; 3: 737-747

15 Kuna ST, Badr MS, Kimoff RJ et al. An official ATS/AASM/ACCP/ERS Workshop report: research priorities in ambulatory management of adults with obstructive sleep apnea. Proc Am Thorac Soc 2011; 8: 1 - 16
16 Ragette $R$, Wang $Y$, Weinreich $G$ et al. Diagnostic performance of single airflow channel recording (ApneaLink) in home diagnosis of sleep apnea. Sleep Breath 2010; 14: 109-114

17 Yadollahi A, Giannouli E, Moussavi Z. Sleep apnea monitoring and diagnosis based on pulse oximetry and tracheal sound signals. Med Biol Eng Comput 2010; 48: 1087-1097

18 Zamarron C, Gude F, Barcala J et al. Utility of oxygen saturation and heart rate spectral analysis obtained from pulse oximetric recordings in the diagnosis of sleep apnea syndrome. Chest 2003; 12: 1567-1576

19 Guilleminault C, Connolly S, Winkle $R$ et al. Cyclical variation of the heart rate in sleep apnoea syndrome. Mechanisms, and usefulness of 24 h electrocardiography as a screening technique. Lancet 1984; 8369: $126-131$

20 Moody GB, Mark RG, Zoccola A et al. Clinical validation of the ECG-derived respiration (EDR) technique. Computers in Cardiology 1986; 13: 507-510

21 Penzel T, McNames J, de Chazal P et al. Systematic comparison of different algorithms for apnoea detection based on electrocardiogram recordings. Med Biol Eng Comput 2002; 40: 402 - 407

22 Kesper K, Canisius S, Penzel T et al. ECG signal analysis for the assessment of sleep-disordered breathing and sleep pattern. Med Biol Eng Comput 2012; 50: 135 - 144

23 Raymond B, Cayton RM, Chappell MJ. Combined index of heart rate variability and oximetry in screening for the sleep apnoea/hypopnoea syndrome. J Sleep Res 2003; 12: 53-61

24 Heneghan C, Chua CP, Garvey JF et al. A portable automated assessment tool for sleep apnea using a combined holter-oximeter. Sleep 2008; 31 : $1432-1439$

25 De Chazal P, Heneghan C, McNicholas WT. Multimodal detection of sleep apnoea using electrocardiogram and oximetry signals. Phil Trans R Soc A 2009; 367: 369-389

26 Chua CP, McDarby G, Heneghan C. Combined electrocardiogram and photoplethysmogram measurements as an indicator of objective sleepiness. Physiol Meas 2008; 29: 857-868

27 Schnall R, Shlitner A, Sheffy J et al. Periodic profound peripheral vasoconstriction - a new marker of obstructive sleep apnea. Sleep 1999; 22: 939-946

28 Bar A, Pillar G, Dvir I et al. Evaluation of a portable device based on peripheral arterial tone for unattended home sleep studies. Chest 2003; 123: $695-703$

29 Senny F, Destine J, Poirrier R. Midsagittal jaw movement analysis for the scoring of sleep apnea and hypopneas. IEEE Trans Biomed Eng 2008; 55: $87-95$

30 Cheliout-Heraut F, Senny F, Djouadi F et al. Obstructive sleep apnoea syndrome: comparison between polysomnography and portable sleep monitoring based on jaw recordings. Neurophysiol Clin 2011; 41: $191-198$

31 Senny F, Destine J, Poirrier R. Midsaggital jaw movements as a sleep/ wake marker. IEEE Trans Biomed Eng 2009; 56: 303-309

32 Pack AI. Sleep medicine: strategies for change. J Clin Sleep Med 2011; 7: $577-579$

33 Penzel T, Fietze I, Hirshkowitz M, Vorstand der DGSM. Diagnostik in der Schlafmedizin. Somnologie 2011; 15: 78-83

34 Bonnet MH, Doghramji K, Roehrs T et al. The scoring of arousal in sleep: reliability, validity, and alternatives. J Clin Sleep Med 2007; 3: 133145

35 Caples SM, Rosen CL, Shen WK et al. The scoring of cardiac events during sleep. J Clin Sleep Med 2007; 3: 147-154

36 Grigg-Damberger M, Gozal D, Marcus CL et al. The visual scoring of sleep and arousal in infants and children. J Clin Sleep Med 2007; 3: 201-240

37 Penzel T, Hirshkowitz M, Harsh J et al. Digital analysis and Technical Specifications. J Clin Sleep Med 2007; 3: 109-120

38 Redline S, Budhiraja R, Kapur Vet al. The scoring of respiratory events in sleep: reliability and validity. J Clin Sleep Med 2007; 3: 169-200

39 Silber MH, Ancoli-Israel S, Bonnet MH et al. The Visual Scoring of Sleep in Adults. J Clin Sleep Med 2007; 3: 121-131

40 Walters AS, Lavigne G, Hening W et al. Movements in sleep: A review by the movement task force of the American academy of sleep medicine.J Clin Sleep Med 2007; 3: 155 -167 